\title{
On the Onsager symmetry of the effective dielectric tensor for plasmas in inhomogeneous magnetic field
}

\author{
R. S. Schneider ${ }^{1}$, L. F. Ziebell ${ }^{1}$, and R. Gaelzer ${ }^{2}$ \\ ${ }^{1}$ Instituto de Física, UFRGS, Caixa Postal 15051, 91501-970 Porto Alegre, RS, Brazil \\ ${ }^{2}$ Instituto de Física e Matemática, UFPel, Caixa Postal 354, 96010-900 Pelotas, RS, Brazil
}

Received on 21 January, 2004; revised version received on 30 April, 2004

\begin{abstract}
The preservation of Onsager symmetry for the effective dielectric tensor is discussed for a homogeneous plasma immersed in a inhomogeneous magnetic field, using the unperturbed orbits correct up to order $k_{B}$, which is the scalelength of the field inhomogeneity. General features of the calculation of the components of the tensor are discussed and detailed calculations are developed for the $z z$ component, which is shown to satisfy the conditions for Onsager symmetry, in agreement with previous results obtained using less precise expressions for the unperturbed orbits.
\end{abstract}

\section{Introduction}

The effective dielectric tensor has been proposed as the correct form to be used for the description of waves in inhomogeneous plasmas in the context of a local approximation [1]. Its basic property is that it is aimed to satisfy energy conservation, even in the presence of inhomogeneities. This property of energy conservation is closely connected with the property of Onsager symmetry, since if Onsager symmetry is satisfied the anti-Hermitian parts of the dielectric tensor only feature resonant parts, properly describing waveparticle energy exchange. On the other hand, if Onsager symmetry is not satisfied, non-resonant terms appear in the anti-Hermitian parts of the dielectric tensor, which describe the variation of the wave amplitude due to the modification of the group velocity in an inhomogeneous medium, not true absorption or amplification [2, 3]. This point has been illustrated with examples in Ref. [4], where results obtained from the dispersion relation with use of the effective dielectric tensor are compared with results obtained using other approaches found in the literature [2,5-8]. The Onsager symmetry of the effective dielectric tensor for electromagnetic waves has also been discussed in Ref. [9], for the case of homogeneous magnetic field and inhomogeneous plasma parameters.

Fur the purpose of the present investigation, it is important to remark that, although the general conception of the effective dielectric tensor is aimed to satisfy energy conservation, the proper symmetry of the tensor may be lost due to approximations introduced in the process of actual calculation of specific expressions of its components. It is therefore important to investigate limiting cases which allow detailed calculation. Here we investigate the case of inhomogeneous magnetic field, neglecting the density inhomogeneity which occurs in order to satisfy equilibrium conditions. More realistic situations which contain simultaneously inhomoge- neities in the magnetic field and in the plasma parameters can of course be treated with the formalism of the effective dielectric tensor. However, since our objective is to discuss specific features related to field inhomogeneity, and improve the accuracy of the calculations as compared to previous approaches, we neglect inhomogeneities in the plasma parameters.

In previous investigations of the effective dielectric tensor for the case of inhomogeneous magnetic field, we have considered high frequency oscillations propagating in arbitrary directions in a plasma, and we have obtained explicit expressions for the components of the effective dielectric tensor, which satisfy Onsager symmetry $[4,10]$. We now return to the subject, aiming to improve the accuracy of the calculations, in the sense that the unperturbed orbits will be described more precisely than in previous work. We consider a homogeneous plasma immersed in a inhomogeneous magnetic field along the $z$ direction, $\mathbf{B}_{0}=B_{0}(x) \mathbf{e}_{z}$, where $B_{0}(x)=B_{0}(0)\left(1+k_{B} x\right)$. We also assume waves propagating in an arbitrary direction relative to the ambient magnetic field and to the field inhomogeneity. Using the proposed geometry we solve the equations of movement and obtain the unperturbed orbits, correct up to order $k_{B}$. In references $[4,10]$, where a dielectric tensor featuring Onsager symmetry was obtained, we had used the unperturbed orbits, but neglecting some corrections of order $k_{B}$, while keeping the correction to the cyclotron frequency $\Omega_{\alpha 0}$, which is necessary in order to avoid secular terms, and the term proportional to $\tau$ in the $y^{\prime}$ coordinate, which describes the drift of the guiding center due to the inhomogeneity. For the present investigation, we keep all the other terms of order $k_{B}$ in the expressions for the unperturbed orbits, re-derive the dielectric tensor, and discuss the ensuing properties of symmetry.

This work presents one example of the calculation which we are developing in order to obtain an effective dielectric tensor exactly correct to order $k_{B}$, to be used in the inves- 
tigation of waves propagating in inhomogeneous plasmas. We develop in detail the procedure which conducts to the $z z$ component of the effective dielectric tensor, in order to point out the problems which must be treated. We also observe that for each component the initial approach must be a little different, but the guiding line is that some expansions are forbidden, in the sense that they can give rise to secular contributions; if secular contributions are introduced, the Onsager symmetry disappears.

\section{The Unperturbed Orbits}

The complete set of unperturbed orbits, given in [4] is

$$
\begin{gathered}
p_{x}^{\prime}(\tau)=p_{\perp} \cos \left(\varphi-\omega_{\alpha} \tau\right) \\
+\frac{k_{B} p_{\perp}^{2}}{2 m_{\alpha} \Omega_{\alpha}}\left[2 \cos \varphi \sin \left(\varphi-\omega_{\alpha} \tau\right)-\sin 2\left(\varphi-\omega_{\alpha} \tau\right)\right] \\
p_{y}^{\prime}(\tau)=p_{\perp} \sin \left(\varphi-\omega_{\alpha} \tau\right)+\frac{k_{B} p_{\perp}^{2}}{2 m_{\alpha} \Omega_{\alpha}} \\
-\frac{k_{B} p_{\perp}^{2}}{2 m_{\alpha} \Omega_{\alpha}}\left[2 \cos \varphi \cos \left(\varphi-\omega_{\alpha} \tau\right)-\cos 2\left(\varphi-\omega_{\alpha} \tau\right)\right] \\
x^{\prime}(\tau)-x=\frac{p_{\perp}}{m_{\alpha} \Omega_{\alpha 0}}\left[\sin \varphi-\sin \left(\varphi-\omega_{\alpha} \tau\right)\right] \\
+\frac{k_{B} p_{\perp}^{2}}{2 m_{\alpha}^{2} \Omega_{\alpha}^{2}}\left[2 \cos \varphi \cos \left(\varphi-\omega_{\alpha} \tau\right)\right. \\
\left.-\frac{1}{2} \cos 2\left(\varphi-\omega_{\alpha} \tau\right)-\frac{1}{2} \cos 2 \varphi-1\right] \\
\frac{p_{\perp}}{m_{\alpha} \Omega_{\alpha 0}}\left[\cos \left(\varphi-\omega_{\alpha} \tau\right)-\cos \varphi\right]+\frac{k_{B} p_{\perp}^{2}}{2 \gamma_{\alpha} m_{\alpha}^{2} \Omega_{\alpha}} \tau \\
+\frac{k_{B} p_{\perp}^{2}}{2 m_{\alpha}^{2} \Omega_{\alpha}^{2}}\left[2 \cos \varphi \sin \left(\varphi-\omega_{\alpha} \tau\right)\right. \\
\left.y^{\prime}(\tau)-y \sin 2\left(\varphi-\omega_{\alpha} \tau\right)-\frac{1}{2} \sin 2 \varphi\right] \\
z^{\prime}(\tau)-z=\frac{p_{\|}}{\gamma_{\alpha} m_{\alpha}} \tau,
\end{gathered}
$$

where

$$
\begin{aligned}
& k_{B}=\left.\frac{1}{B_{0}} \frac{d B_{0}}{d x}\right|_{x=0}, B_{0}(x)=B_{0}\left(1+k_{B} x\right), \\
& \omega_{\alpha}=\frac{\Omega_{\alpha 0}}{\gamma_{\alpha}}=\frac{\Omega_{\alpha}}{\gamma_{\alpha}}\left(1+k_{B} x+k_{B} \frac{p_{\perp} \sin \varphi}{m_{\alpha} \Omega_{\alpha}}\right) .
\end{aligned}
$$

These orbit equations satisfy all the necessary conditions. They satisfy the initial conditions, they are selfconsistent and, up to order $k_{B}$, they satisfy the constants of motion.

\section{The zz Component of the Effective Dielectric Tensor}

To obtain the effective dielectric tensor it is necessary to start with the calculation of the tensor $\stackrel{\leftrightarrow}{\varepsilon^{0}}$, which is obtained using the usual expressions obtained with plane wave approximation, but incorporating effects due to the inhomogeneity:

$$
\begin{gathered}
\overleftrightarrow{\varepsilon^{0}}=\overleftrightarrow{1}-i \sum_{\alpha} \frac{4 \pi q_{\alpha}^{2}}{\omega m_{\alpha}} \int d^{3} p \frac{\mathbf{p}}{\gamma_{\alpha}} \mathbf{A}_{\alpha}, \\
\mathbf{A}_{\alpha}=\int_{-\infty}^{0} d \tau \mathbf{O}_{\alpha} e^{i\left[\mathbf{k} \cdot\left(\mathbf{r}^{\prime}-\mathbf{r}\right)-\omega \tau\right]}, \\
\mathbf{O}_{\alpha}=\left(1-\frac{\mathbf{k} \cdot \mathbf{p}^{\prime}}{\gamma_{\alpha} m_{\alpha} \omega}\right) \frac{\partial f_{\alpha 0}}{\partial \mathbf{p}^{\prime}}+\frac{\left(\mathbf{k} \cdot \nabla_{\mathbf{p}^{\prime}}\right) f_{\alpha 0}}{\gamma_{\alpha} m_{\alpha} \omega} \mathbf{p}^{\prime} .
\end{gathered}
$$

As mentioned in the Introduction, for the present application we calculate only the $z z$ component of the tensor. In order to simplify the analysis we consider propagation in the $x-z$ plane. Then $\mathbf{k}=k_{\perp} \mathbf{e}_{\mathbf{x}}+k_{\|} \mathbf{e}_{\mathbf{z}}$. In this case we can write as follows the $z z$ component of the effective tensor

$$
\begin{gathered}
\varepsilon_{z z}(x, \mathbf{k}, \omega)=\frac{1}{2 \pi} \int_{-\infty}^{+\infty} d k_{\perp}^{\prime} \int_{-\infty}^{+\infty} d \eta \\
\times \varepsilon_{z z}^{0}\left(x+\frac{\eta}{2}, k_{\perp}^{\prime}, k_{\|}, \omega\right) e^{i\left(k_{\perp}^{\prime}-k_{\perp}\right) \eta} .
\end{gathered}
$$

We note that some quantities appearing in the integrand of $\varepsilon_{z z}^{0}, z z$ component of the tensor obtained with the plane wave approximation, can be written as follows:

1.

$$
\begin{gathered}
O_{\alpha z}=\frac{\partial f_{\alpha 0}}{\partial p_{\|}}+\frac{\Omega_{\alpha}}{\gamma_{\alpha} \omega} b_{\alpha} L\left(f_{\alpha 0}\right) \cos \left(\varphi-\omega_{\alpha} \tau\right) \\
+\frac{\Omega_{\alpha}}{\gamma_{\alpha} \omega} b_{\alpha} L\left(f_{\alpha 0}\right) \eta_{\alpha}\left[2 \cos \varphi \sin \left(\varphi-\omega_{\alpha} \tau\right)-\sin 2\left(\varphi-\omega_{\alpha} \tau\right)\right]
\end{gathered}
$$

where

$$
\begin{gathered}
L \equiv \frac{p_{\|}}{p_{\perp}} \frac{\partial}{\partial p_{\perp}}-\frac{\partial}{\partial p_{\|}} \\
b_{\alpha} \equiv \frac{k_{\perp} p_{\perp}}{m_{\alpha} \Omega_{\alpha}}, \quad \eta_{\alpha} \equiv \frac{1}{2} \frac{k_{B} p_{\perp}}{m_{\alpha} \Omega_{\alpha}},
\end{gathered}
$$


2.

$$
e^{i\left[\mathbf{k} \cdot\left(\mathbf{r}^{\prime}-\mathbf{r}\right)-\omega \tau\right]}=F_{1}(\varphi, \tau) F_{2}(\varphi) \exp \left[i\left(\frac{k_{\|} p_{\|}}{\gamma_{\alpha} m_{\alpha}}-\omega\right) \tau\right]
$$

where

$$
\begin{gathered}
F_{1}(\varphi, \tau)=\exp \left\{-i b_{\alpha} \frac{\Omega_{\alpha}}{\Omega_{\alpha 0}} \sin \left(\varphi-\omega_{\alpha} \tau\right)\right. \\
\left.+i b_{\alpha} \eta_{\alpha}\left[2 \cos \varphi \cos \left(\varphi-\omega_{\alpha} \tau\right)-\frac{1}{2} \cos 2\left(\varphi-\omega_{\alpha} \tau\right)\right]\right\}, \\
F_{2}(\varphi)=\exp \left\{i b_{\alpha} \frac{\Omega_{\alpha}}{\Omega_{\alpha 0}} \sin \varphi+i b_{\alpha}\left[\frac{1}{2} \cos 2 \varphi+1\right]\right\} .
\end{gathered}
$$

3.

$$
A_{\alpha z}=F_{2}(\varphi) \int_{-\infty}^{0} d \tau \exp \left[i\left(\frac{k_{\|} p_{\|}}{\gamma_{\alpha} m_{\alpha}}-\omega\right) \tau\right]\left(\frac{\partial f_{\alpha 0}}{\partial p_{\|}}-i \frac{L\left(f_{\alpha 0}\right)}{\omega} \frac{\partial}{\partial \tau}\right) F_{1}(\varphi, \tau)
$$

which can be cast in the form

$$
A_{\alpha z}=F_{2}(\varphi) \frac{p_{\|}}{p_{\perp}} \mathcal{L}\left(f_{\alpha 0}\right) \int_{-\infty}^{0} d \tau \exp \left[i\left(\frac{k_{\|} p_{\|}}{\gamma_{\alpha} m_{\alpha}}-\omega\right) \tau\right] F_{1}(\varphi, \tau)-i \frac{L\left(f_{\alpha 0}\right)}{\omega}
$$

where

$$
\mathcal{L} \equiv\left(1-\frac{k_{\|} p_{\|}}{\gamma_{\alpha} m_{\alpha} \omega}\right) \frac{\partial}{\partial p_{\perp}}+\frac{k_{\|} p_{\perp}}{\gamma_{\alpha} m_{\alpha} \omega} \frac{\partial}{\partial p_{\|}}
$$

We observe that $\Omega_{\alpha 0}$ depends on $\varphi$ and, because at some moment it will be necessary to perform the integration in this variable, we must explicitly show this dependence. To this end we approximate

$$
\frac{\Omega_{\alpha}}{\Omega_{\alpha 0}}=\frac{1}{1+k_{B} x+2 \eta_{\alpha} \sin \varphi} \simeq \delta_{x}^{-}-2 \eta_{\alpha} \sin \varphi,
$$

where we define

$$
\delta_{x}^{ \pm} \equiv 1 \pm k_{B} x
$$

Then, we can write

$$
\begin{gathered}
F_{1}(\varphi, \tau)=\exp \left[-i b_{\alpha} \delta_{x}^{-} \sin \left(\varphi-\omega_{\alpha} \tau\right)\right. \\
\left.-\frac{i}{2} b_{\alpha} \eta_{\alpha} \cos 2\left(\varphi-\omega_{\alpha} \tau\right)+2 i b_{\alpha} \eta_{\alpha} \cos \left(\omega_{\alpha} \tau\right)\right] \\
F_{2}(\varphi)=\exp \left[i b_{\alpha} \delta_{x}^{-} \sin \varphi+\frac{i}{2} b_{\alpha} \eta_{\alpha} \cos 2 \varphi-2 i b_{\alpha} \eta_{\alpha}\right] .
\end{gathered}
$$

Using one of the definitions of the Generalized Bessel Functions introduced in references $[11,12,13]$

$$
e^{ \pm i x \sin \phi \pm i y \cos 2 \phi}=\sum_{n=-\infty}^{\infty} e^{ \pm i n \phi} J_{n}(x, y ; \pm i)
$$

and

$$
e^{ \pm i x \cos \alpha}=\sum_{l=-\infty}^{\infty} I_{l}( \pm i x) e^{i l \alpha}
$$

we can write

$$
F_{1}(\varphi, \tau)=\sum_{l, n=-\infty}^{+\infty} J_{n}\left(b_{\alpha} \delta_{x}^{-}, \frac{1}{2} b_{\alpha} \eta_{\alpha} ;-i\right)
$$

$$
\times I_{l}\left(2 i b_{\alpha} \eta_{\alpha}\right) e^{-i n \varphi} e^{i(l+n) \omega_{\alpha} \tau}
$$

and

$$
A_{\alpha z}=\frac{\gamma_{\alpha}}{\omega} F_{2}(\varphi) \frac{p_{\|}}{p_{\perp}} \mathcal{L}\left(f_{\alpha 0}\right) \sum_{l, n=-\infty}^{+\infty} J_{n}\left(b_{\alpha} \delta_{x}^{-}, \frac{1}{2} b_{\alpha} \eta_{\alpha} ;-i\right)
$$

$$
\times I_{l}\left(2 i b_{\alpha} \eta_{\alpha}\right) e^{-i n \varphi} \int_{0}^{\infty} d \tau e^{i \mathcal{D}_{(l+n) \alpha} \tau}-i \frac{L\left(f_{\alpha 0}\right)}{\omega}
$$

where the resonance term is defined by

$$
\mathcal{D}_{n \alpha} \equiv \gamma_{\alpha}-\frac{k_{\|} p_{\|}}{\omega m_{\alpha}}-n \frac{\Omega_{\alpha}}{\omega}\left(\delta_{x}^{+}+2 \eta_{\alpha} \sin \varphi\right)
$$

In order to obtain $\varepsilon_{z z}^{0}$ the last step is to perform the $\varphi$ integration in the momentum integration:

$$
\mathcal{I}_{\varphi} \equiv \int_{0}^{2 \pi} d \varphi F_{2}(\varphi) e^{-i n \varphi} e^{i \mathcal{D}_{(l+n) \alpha} \tau}
$$

which gives

$$
\mathcal{I}_{\varphi}=2 \pi e^{i D_{(l+n) \alpha} \tau} e^{-2 i b_{\alpha} \eta_{\alpha}} J_{n}\left(b_{\alpha} \delta_{x}^{-}-\alpha_{l+n} \tau, \frac{1}{2} b_{\alpha} \eta_{\alpha} ; i\right)
$$

where

$$
\begin{gathered}
D_{n \alpha} \equiv \gamma_{\alpha}-\frac{k_{\|} p_{\|}}{\omega m_{\alpha}}-n \frac{\Omega_{\alpha}}{\omega} \delta_{x}^{+}, \\
\alpha_{n} \equiv 2 n \eta_{\alpha} \frac{\Omega_{\alpha}}{\omega} .
\end{gathered}
$$


Finally we obtain

$$
\begin{gathered}
\varepsilon_{z z}^{0}=1-i \sum_{\alpha} \frac{4 \pi q_{\alpha}^{2}}{\omega^{2} m_{\alpha}} \sum_{l, n=-\infty}^{+\infty} \\
\times \int d^{3} p \frac{p_{\|}^{2}}{p_{\perp}} \mathcal{L}\left(f_{\alpha 0}\right) \mathcal{H}\left(2 i b_{\alpha} \eta_{\alpha}\right) \int_{0}^{\infty} d \tau e^{i D_{(l+n) \alpha} \tau}
\end{gathered}
$$

$$
\begin{aligned}
\times J_{n}\left(b_{\alpha} \delta_{x}^{-}\right. & \left.-\alpha_{l+n} \tau, \frac{1}{2} b_{\alpha} \eta_{\alpha} ; i\right) J_{n}\left(b_{\alpha} \delta_{x}^{-}, \frac{1}{2} b_{\alpha} \eta_{\alpha} ;-i\right) \\
& -\sum_{\alpha} \frac{4 \pi q_{\alpha}^{2}}{\omega^{2} m_{\alpha}} \int d^{3} p \frac{p_{\|}}{\gamma_{\alpha}} L\left(f_{\alpha 0}\right),
\end{aligned}
$$

where $\mathcal{H}_{l}(z) \equiv e^{-z} I_{l}(z)$.

In order to perform the BGI correction in this component of the tensor, we write equation (10) as

$$
\begin{gathered}
\varepsilon_{z z}(x, \mathbf{k}, \omega)=1-\sum_{\alpha} \frac{4 \pi q_{\alpha}^{2}}{\omega^{2} m_{\alpha}} \int d^{3} p \frac{p_{\|}}{\gamma_{\alpha}} L\left(f_{\alpha 0}\right) \\
-\frac{i}{2 \pi} \sum_{\alpha} \frac{4 \pi q_{\alpha}^{2}}{\omega^{2} m_{\alpha}} \sum_{l, n} \int_{0}^{\infty} d \tau \int d^{3} p \frac{p_{\|}^{2}}{p_{\perp}} \mathcal{L}\left(f_{\alpha 0}\right) e^{i D_{(l+n) \alpha}(x) \tau} \mathcal{I}_{B G I},
\end{gathered}
$$

where

$$
\begin{gathered}
\mathcal{I}_{B G I} \equiv \int_{-\infty}^{+\infty} d k_{\perp}^{\prime} \int_{-\infty}^{+\infty} d \eta e^{i\left[k_{\perp}^{\prime}-k_{\perp}-(l+n) k_{B} \Omega_{\alpha} \tau / 2 \omega\right] \eta} \mathcal{H}_{l}\left(2 i b_{\alpha}^{\prime} \eta_{\alpha}\right) \\
\times J_{n}\left(b_{\alpha}^{\prime} \delta_{x+\eta / 2}^{-}-\alpha_{l+n} \tau, \frac{1}{2} b_{\alpha}^{\prime} \eta_{\alpha} ; i\right) J_{n}\left(b_{\alpha}^{\prime} \delta_{x+\eta / 2}^{-}, \frac{1}{2} b_{\alpha}^{\prime} \eta_{\alpha} ;-i\right)
\end{gathered}
$$

Now we use in this expression the integral representation of the Generalized Bessel Functions, given by

$$
J_{n}(x, y ; \pm i)=\frac{1}{2 \pi} \int_{0}^{2 \pi} d \phi e^{ \pm i x \sin \phi \pm i y \cos 2 \phi \mp i n \phi} .
$$

We obtain

$$
\begin{aligned}
& \mathcal{I}_{B G I}=\frac{1}{(2 \pi)^{2}} \int_{0}^{2 \pi} d \phi_{1} \int_{0}^{2 \pi} d \phi_{2} \int_{-\infty}^{+\infty} d k_{\perp}^{\prime} \mathcal{H}_{l}\left(2 i b_{\alpha}^{\prime} \eta_{\alpha}\right) \\
& \times \exp \left[i\left(b_{\alpha}^{\prime} \delta_{x}^{-}-\alpha_{l+n} \tau\right) \sin \phi_{1}+\frac{i}{2} b_{\alpha}^{\prime} \eta_{\alpha} \cos 2 \phi_{1}-i n \phi_{1}\right] \\
& \quad \times \exp \left[-i b_{\alpha}^{\prime} \delta_{x}^{-} \sin \phi_{2}-\frac{i}{2} b_{\alpha}^{\prime} \eta_{\alpha} \cos 2 \phi_{2}+i n \phi_{2}\right] \\
& \times \int_{-\infty}^{+\infty} d \eta e^{i\left\{\left[1-\eta_{\alpha}\left(\sin \phi_{1}-\sin \phi_{2}\right)\right] k_{\perp}^{\prime}-k_{\perp}-(l+n) k_{B} \Omega_{\alpha} \tau / 2 \omega\right\} \eta} .
\end{aligned}
$$

Performing the $\eta$ integration

$$
\begin{gathered}
\mathcal{I}_{B G I}=\frac{1}{2 \pi} \int_{0}^{2 \pi} d \phi_{1} \int_{0}^{2 \pi} d \phi_{2} \frac{1}{1-\eta_{\alpha}\left(\sin \phi_{1}-\sin \phi_{2}\right)} \\
\times \int_{-\infty}^{+\infty} d b_{\alpha}^{\prime} \mathcal{H}_{l}\left(2 i b_{\alpha}^{\prime} \eta_{\alpha}\right) \\
\times \exp \left[i\left(b_{\alpha}^{\prime} \delta_{x}^{-}-\alpha_{l+n} \tau\right) \sin \phi_{1}+\frac{i}{2} b_{\alpha}^{\prime} \eta_{\alpha} \cos 2 \phi_{1}-i n \phi_{1}\right] \\
\times \exp \left[-i b_{\alpha}^{\prime} \delta_{x}^{-} \sin \phi_{2}-\frac{i}{2} b_{\alpha}^{\prime} \eta_{\alpha} \cos 2 \phi_{2}+i n \phi_{2}\right] \\
\times \delta\left(b_{\alpha}^{\prime}-\frac{b_{\alpha}+\alpha_{l+n} \tau / 2}{1-\eta_{\alpha}\left(\sin \phi_{1}-\sin \phi_{2}\right)}\right) .
\end{gathered}
$$

Performing the $b_{\alpha}^{\prime}$ integration

$$
\begin{aligned}
& \mathcal{I}_{B G I}= \frac{1}{2 \pi} \int_{0}^{2 \pi} d \phi_{1} \int_{0}^{2 \pi} d \phi_{2} \frac{1}{1-\eta_{\alpha}\left(\sin \phi_{1}-\sin \phi_{2}\right)} \\
& \times \mathcal{H}_{l}\left(2 i \frac{b_{\alpha}+\alpha_{l+n} \tau / 2}{1-\eta_{\alpha}\left(\sin \phi_{1}-\sin \phi_{2}\right)} \eta_{\alpha}\right) \\
& \times \exp \left[i\left(\frac{b_{\alpha}+\alpha_{l+n} \tau / 2}{1-\eta_{\alpha}\left(\sin \phi_{1}-\sin \phi_{2}\right)} \delta_{x}^{-}-\alpha_{l+n} \tau\right) \sin \phi_{1}\right. \\
&\left.+\frac{i}{2} \frac{b_{\alpha}+\alpha_{l+n} \tau / 2}{1-\eta_{\alpha}\left(\sin \phi_{1}-\sin \phi_{2}\right)} \eta_{\alpha} \cos 2 \phi_{1}-i n \phi_{1}\right] \\
& \times \exp \left[-i\left(\frac{b_{\alpha}+\alpha_{l+n} \tau / 2}{1-\eta_{\alpha}\left(\sin \phi_{1}-\sin \phi_{2}\right)}\right) \delta_{x}^{-} \sin \phi_{2}\right. \\
&\left.-\frac{i}{2} \frac{b_{\alpha}+\alpha_{l+n} \tau / 2}{1-\eta_{\alpha}\left(\sin \phi_{1}-\sin \phi_{2}\right)} \eta_{\alpha} \cos 2 \phi_{2}+i n \phi_{2}\right] .
\end{aligned}
$$

We now expand the different factors of this expression, maintaining all terms up to order $k_{B}$; then

$$
\begin{gathered}
\mathcal{I}_{B G I} \simeq \frac{1}{2 \pi} \int_{0}^{2 \pi} d \phi_{1} \int_{0}^{2 \pi} d \phi_{2}\left[1+\eta_{\alpha}\left(\sin \phi_{1}-\sin \phi_{2}\right)\right] \\
\times \mathcal{H}_{l}\left(2 i b_{\alpha} \eta_{\alpha}\right) \\
\times \exp \left[i\left(\beta_{\alpha(l+n)}^{-}+b_{\alpha} \eta_{\alpha}\left(\sin \phi_{1}-\sin \phi_{2}\right)\right) \sin \phi_{1}\right. \\
\left.+\frac{i}{2} b_{\alpha} \eta_{\alpha} \cos 2 \phi_{1}-i n \phi_{1}\right] \\
\times \exp \left[-i\left(\beta_{\alpha(l+n)}^{+}+b_{\alpha} \eta_{\alpha}\left(\sin \phi_{1}-\sin \phi_{2}\right)\right) \sin \phi_{2}\right.
\end{gathered}
$$




$$
\left.-\frac{i}{2} b_{\alpha} \eta_{\alpha} \cos 2 \phi_{2}+i n \phi_{2}\right]
$$

where

$$
\beta_{\alpha n}^{ \pm} \equiv b_{\alpha} \delta_{x}^{-} \pm \alpha_{n} \tau / 2
$$

We also use, keeping only terms linear in $k_{B}$

$$
\mathcal{H}_{l}\left(2 i b_{\alpha} \eta_{\alpha}\right) \simeq\left(1-i b_{\alpha} \eta_{\alpha}\right) \delta_{l, 0}+i b_{\alpha} \eta_{\alpha} \delta_{|l|, 1}
$$

which, after some trigonometric manipulations gives

$$
\begin{gathered}
\mathcal{I}_{B G I} \simeq \frac{1}{2 \pi}\left[\left(1-i b_{\alpha} \eta_{\alpha}\right) \delta_{l, 0}+i b_{\alpha} \eta_{\alpha} \delta_{|l|, 1}\right] \\
\times \int_{0}^{2 \pi} d \phi_{1} \int_{0}^{2 \pi} d \phi_{2}\left[1+\eta_{\alpha}\left(\sin \phi_{1}-\sin \phi_{2}\right)-2 i b_{\alpha} \eta_{\alpha} \sin \phi_{1} \sin \phi_{2}-i b_{\alpha} \eta_{\alpha} \cos 2 \phi_{2}\right] \\
\times \exp \left[i \beta_{\alpha(l+n)}^{-} \sin \phi_{1}-i n \phi_{1}\right] \exp \left[-i \beta_{\alpha(l+n)}^{+} \sin \phi_{2}+i n \phi_{2}\right] .
\end{gathered}
$$

Using the following integral representations of the usual Bessel functions and its derivatives

$$
\begin{gathered}
2 \pi J_{n}(z)=\int_{0}^{2 \pi} d \phi e^{ \pm i z \sin \phi \mp i n \phi} \\
\mp 2 i \pi J_{n}^{\prime}(z)=\int_{0}^{2 \pi} d \phi \sin \phi e^{ \pm i z \sin \phi \mp i n \phi} \\
2 \pi\left[2\left(\frac{n^{2}}{z^{2}}-\frac{1}{2}\right) J_{n}(z)-2 \frac{J_{n}^{\prime}(z)}{z}\right]=\int_{0}^{2 \pi} d \phi \cos 2 \phi e^{ \pm i z \sin \phi \mp i n \phi},
\end{gathered}
$$

we obtain, maintaining in the coefficients of the Bessel functions and its derivative terms up to order $k_{B}$,

Using

$$
\begin{gathered}
\mathcal{I}_{B G I} \simeq 2 \pi \delta_{l, 0}\left\{J_{n}\left(\beta_{\alpha(l+n)}^{-}\right) J_{n}\left(\beta_{\alpha(l+n)}^{+}\right)-i \eta_{\alpha}\left[J_{n}^{\prime}\left(\beta_{\alpha(l+n)}^{-}\right) J_{n}\left(\beta_{\alpha(l+n)}^{+}\right)-J_{n}\left(\beta_{\alpha(l+n)}^{-}\right) J_{n}^{\prime}\left(\beta_{\alpha(l+n)}^{+}\right)\right]\right\} \\
-2 \pi \delta_{l, 0}\left\{2 i b_{\alpha} \eta_{\alpha} J_{n}^{\prime}\left(\beta_{\alpha(l+n)}^{-}\right) J_{n}^{\prime}\left(\beta_{\alpha(l+n)}^{+}\right)+2 i b_{\alpha} \eta_{\alpha} \frac{n^{2}}{b_{\alpha}^{2}} J_{n}\left(\beta_{\alpha(l+n)}^{-}\right) J_{n}\left(\beta_{\alpha(l+n)}^{+}\right)\right\} \\
+2 \pi i b_{\alpha} \eta_{\alpha} \delta_{|l|, 1} J_{n}\left(\beta_{\alpha(l+n)}^{-}\right) J_{n}\left(\beta_{\alpha(l+n)}^{+}\right) .
\end{gathered}
$$

$$
\begin{gathered}
\sum_{n=-\infty}^{+\infty} \sum_{l= \pm 1} J_{n}\left(\beta_{\alpha(l+n)}^{-}\right) J_{n}\left(\beta_{\alpha(l+n)}^{+}\right) e^{i D_{(l+n) \alpha} \tau} \\
=\sum_{n=-\infty}^{+\infty}\left[J_{n-1}\left(\beta_{\alpha n}^{-}\right) J_{n-1}\left(\beta_{\alpha n}^{+}\right)+J_{n+1}\left(\beta_{\alpha n}^{-}\right) J_{n+1}\left(\beta_{\alpha n}^{+}\right)\right] e^{i D_{n \alpha} \tau}
\end{gathered}
$$

we can obtain

$$
\begin{aligned}
& \frac{1}{2 \pi} \sum_{l, n=-\infty}^{+\infty} \mathcal{I}_{B G I} e^{i D_{(l+n) \alpha} \tau}=\sum_{n=-\infty}^{+\infty}\left\{J_{n}\left(\beta_{\alpha n}^{-}\right) J_{n}\left(\beta_{\alpha n}^{+}\right)\right. \\
& \left.-i \eta_{\alpha}\left[J_{n}^{\prime}\left(\beta_{\alpha n}^{-}\right) J_{n}\left(\beta_{\alpha n}^{+}\right)-J_{n}\left(\beta_{\alpha n}^{-}\right) J_{n}^{\prime}\left(\beta_{\alpha n}^{+}\right)\right]\right\} e^{i D_{n \alpha} \tau},
\end{aligned}
$$

which gives for the $z z$ component of the effective dielectric tensor

$$
\begin{gathered}
\varepsilon_{z z}(x, \mathbf{k}, \omega)=1-\sum_{\alpha} \frac{4 \pi q_{\alpha}^{2}}{\omega^{2} m_{\alpha}} \int d^{3} p \frac{p_{\|}}{\gamma_{\alpha}} L\left(f_{\alpha 0}\right) \\
-\frac{i}{2 \pi} \sum_{\alpha} \frac{4 \pi q_{\alpha}^{2}}{\omega^{2} m_{\alpha}} \sum_{-\infty}^{+\infty} \int_{0}^{\infty} d \tau \int d^{3} p \frac{p_{\|}^{2}}{p_{\perp}} \mathcal{L}\left(f_{\alpha 0}\right) e^{i D_{n \alpha} \tau} \\
\times\left\{J_{n}\left(\beta_{\alpha n}^{-}\right) J_{n}\left(\beta_{\alpha n}^{+}\right)-i \eta_{\alpha}\left[J_{n}^{\prime}\left(\beta_{\alpha n}^{-}\right) J_{n}\left(\beta_{\alpha n}^{+}\right)-J_{n}\left(\beta_{\alpha n}^{-}\right) J_{n}^{\prime}\left(\beta_{\alpha n}^{+}\right)\right]\right\} .
\end{gathered}
$$




\section{The Onsager Symmetry}

For the $z z$ component of the dielectric tensor the symmetry condition to be verified is

$$
\begin{gathered}
\varepsilon_{z z}\left(\mathbf{k}, \omega ; \mathbf{B}_{0},\left\{f_{\alpha 0}\left(p_{\perp}^{2}, p_{\|}\right)\right\}\right) \\
=\varepsilon_{z z}\left(-\mathbf{k}, \omega ;-\mathbf{B}_{0},\left\{f_{\alpha 0}\left(p_{\perp}^{2},-p_{\|}\right)\right\}\right) .
\end{gathered}
$$

What we must do in the expressions here obtained, in order to construct the right-hand side of the symmetry condition, is to make the substitutions

$$
\begin{aligned}
& \Omega_{\alpha} \rightarrow-\Omega_{\alpha}, \\
& k_{B} \rightarrow k_{B}, \\
& b_{\alpha} \rightarrow b_{\alpha}, \\
& \eta_{\alpha} \rightarrow-\eta_{\alpha}, \\
& \delta_{x}^{ \pm} \rightarrow \delta_{x}^{ \pm}, \\
& \alpha_{(l+n)} \rightarrow \alpha_{(l+n)}, \\
& \beta_{\alpha n}^{ \pm} \rightarrow \beta_{\alpha n}^{ \pm}, \\
& L \rightarrow L, \\
& \mathcal{L} \rightarrow \overline{\mathcal{L}}=\left(\begin{array}{l}
k_{\|} p_{\|} \\
\gamma_{\alpha} m_{\alpha} \omega
\end{array}\right) \frac{\partial}{\partial p_{\perp}}+\frac{k_{\|} p_{\perp}}{\gamma_{\alpha} m_{\alpha} \omega} \frac{\partial}{\partial p_{\|}} \\
& D_{(l+n) \alpha} \rightarrow \bar{D}_{(l+n) \alpha}=\gamma_{\alpha}+\frac{k_{\|} p_{\|}}{\omega m_{\alpha}}+(l+n) \frac{\Omega_{\alpha}}{\omega} \delta_{x}^{+} .
\end{aligned}
$$

We also need to change the dummy variables $p_{\|} \rightarrow-p_{\|}$, $n \rightarrow-n$ and $l \rightarrow-l$, which produces

$$
\begin{aligned}
f_{\alpha 0}\left(p_{\perp}^{2},-p_{\|}\right) & \rightarrow f_{\alpha 0}\left(p_{\perp}^{2},-p_{\|}\right), \\
\alpha_{(l+n)} & \rightarrow-\alpha_{(l+n)}, \\
\beta_{\alpha n}^{ \pm} & \rightarrow \beta_{\alpha n}^{\mp}, \\
L & \rightarrow-L, \\
\overline{\mathcal{L}} & \rightarrow \mathcal{L}, \\
\bar{D}_{(l+n) \alpha} & \rightarrow D_{(l+n) \alpha} .
\end{aligned}
$$

It is trivial to see that the $z z$ component of the effective dielectric tensor satisfies the required Onsager's symmetry. The $z z$ component of the dielectric tensor without the BGI correction doesn't feature this symmetry, since after the substitutions described above it transforms into

$$
\begin{gathered}
\varepsilon_{z z}^{0} \rightarrow 1-i \sum_{\alpha} \frac{4 \pi q_{\alpha}^{2}}{\omega^{2} m_{\alpha}} \sum_{l, n=-\infty}^{+\infty}(-1)^{l} \\
\times \int d^{3} p \frac{p_{\|}^{2}}{p_{\perp}} \mathcal{L}\left(f_{\alpha 0}\left(p_{\perp}^{2}, p_{\|}\right)\right) \mathcal{H}_{l}\left(-2 i b_{\alpha} \eta_{\alpha}\right) \int_{0}^{\infty} d \tau e^{i D_{(l+n) \alpha} \tau} \\
\times J_{n}\left(b_{\alpha} \delta_{x}^{-}+\alpha_{l+n} \tau, \frac{1}{2} b_{\alpha} \eta_{\alpha} ;-i\right) J_{n}\left(b_{\alpha} \delta_{x}^{-}, \frac{1}{2} b_{\alpha} \eta_{\alpha} ; i\right)
\end{gathered}
$$

$$
-\sum_{\alpha} \frac{4 \pi q_{\alpha}^{2}}{\omega^{2} m_{\alpha}} \int d^{3} p \frac{p_{\|}}{\gamma_{\alpha}} L\left(f_{\alpha 0}\left(p_{\perp}^{2}, p_{\|}\right)\right)
$$

which is not the same as the expression given by equation (18).

These calculations show that the $z z$ component of the effective dielectric tensor in the case of magnetic field with inhomogeneity perpendicular to the direction of the field satisfies Onsager symmetry, when all terms of the order of the inhomogeneity parameter are kept in the expressions for the unperturbed orbits of the particles. As we have seen, the derivation of the dielectric tensor in this case requires a large amount of mathematical manipulations, considerably more than in the case of more approximated orbits. They certainly require much heavier load of algebraic manipulations than the homogeneous case. Similar calculations must be now developed for the other components of the effective dielectric tensor, in order to verify if Onsager symmetry is indeed satisfied by the tensor, as demonstrated in the case of simpler expressions for the unperturbed orbits $[4,10]$, as well as in the case of homogeneous field and inhomogeneous plasma parameters [14].

\section{References}

[1] V. S. Beskin, A. V. Gurevich, and Y. I. Istomin, Sov. Phys. JETP 65, 715 (1987).

[2] C. N. Lashmore-Davies and R. O. Dendy, Phys. Fluids B 4, 493 (1992).

[3] D. C. McDonald, R. A. Cairns, and C. N. Lashmore-Davies, Phys. Plasmas 1, 842 (1994).

[4] R. Gaelzer, R. S. Schneider, and L. F. Ziebell, Phys. Rev. E 51, 2407 (1995).

[5] A. B. Mikhailovskii and O. G. Onishchenko, J. Plasma Phys. 37, 15 (1987).

[6] C. N. Lashmore-Davies and R. O. Dendy, Phys. Fluids B 1, 1565 (1989).

[7] C. N. Lashmore-Davies and R. O. Dendy, Phys. Rev. Lett. 62, 1982 (1989).

[8] R. A. Cairns et al., Phys. Fluids B 3, 2953 (1991).

[9] M. Nambu, Phys. Plasmas 3, 4325 (1996).

[10] R. Gaelzer, R. S. Schneider, and L. F. Ziebell, Phys. Rev. E 55, 5859 (1997).

[11] G. Dattoli, L. Gianessi, L. Mezi, and A. Torre, Il Nuovo Cimento 105B, 327 (1990).

[12] G. Dattoli et al., Il Nuovo Cimento 106B, 21 (1991).

[13] 1. Galué, H. G. Khajah, and S. L. Kalla, J. Comp. Ap. Mathem. 118, 143 (2000).

[14] R. A. Caldela Filho, R. S. Schneider, and L. F. Ziebell, J. Plasma Phys. 42, 165 (1989). 